\title{
Elevated Monoamine Oxidase A Binding During Major Depressive Episodes Is Associated with Greater Severity and Reversed Neurovegetative Symptoms
}

\author{
Lina Chiuccariello', Sylvain Houle', Laura Miler', Robert G Cooke', Pablo M Rusjan', Grazyna Rajkowska², \\ Robert D Levitan', Stephen J Kish', Nathan J Kolla', Xiaoming Oư', Alan A Wilson' and Jeffrey H Meyer*,' \\ 'CAMH Research Imaging Centre and Campbell Family Mental Health Research Institute, Centre for Addiction and Mental Health, Department \\ of Psychiatry, Pharmacology and Toxicology, Institute of Medical Sciences, University of Toronto, Toronto, Ontario, Canada; ${ }^{2}$ Department of \\ Psychiatry and Human Behavior, University of Mississippi Medical Center, Jackson, Mississippi, USA
}

\begin{abstract}
Inadequate treatment response occurs in approximately 40\% of major depressive episodes (MDEs), and one approach to solve this is careful matching of treatment to the specific pathologies of MDE. One such biological abnormality is elevated monoamine oxidase A (MAO-A) levels, which occurs in the prefrontal and anterior cingulate cortex (PFC and ACC) during MDE; however, the subtypes for which this abnormality is most prominent are unknown. We hypothesized that MAO-A levels in the PFC and ACC are most elevated in MDE with greater severity and reversed neurovegetative symptoms (hypersomnia and either hyperphagia or weight gain). MAO-A $V_{T}$ (an index of MAO-A density) was measured using [ ' $\mathrm{C}$ ]harmine positron emission tomography (PET) in 42 subjects with MDEs secondary to major depressive disorder and 37 healthy controls. The effect of severity and reversed neurovegetative symptoms on MAO-A $V_{T}$ in the PFC and ACC was analyzed using a multivariate analysis of variance (MANOVA). Greater severity and reversed neurovegetative symptoms were associated with elevated MAO-A $V_{T}$ in the PFC and ACC (MANOVA, severity: $F_{(2,38)}=5.44$, $p=0.008$; reversed neurovegetative symptoms: $F_{(2,38)}=5.13, p=0.0 \mathrm{I}$ ). Increased MAO-A level, when greater severity and reversed neurovegetative symptoms are present, may explain the association of these clinical features with a preferential response to $M A O$ inhibitors, which is especially well-evidenced for reversed neurovegetative symptoms in MDE. As MAO-A creates oxidative stress, facilitates apoptosis, and metabolizes monoamines, therapeutics opposing these processes are predicted to best treat MDE with greater severity and reversed neurovegetative symptoms.

Neuropsychopharmacology (20 I4) 39, 973-980; doi:I0.1038/npp.2013.297; published online I3 November 20I3
\end{abstract}

Keywords: oxidative stress; apoptosis; serotonin; monoamine oxidase; major depressive disorder

\section{INTRODUCTION}

Major depressive disorder (MDD) is the leading cause of death and disability in moderate to high income countries (WHO, 2008). A key reason for this is treatment resistance, as fewer than $40 \%$ of major depressive episodes (MDEs) have an optimal response to initial treatment (Trivedi et al, 2006). One strategy to improve treatment response is to apply clinical measures or biomarkers to identify pathological subtypes so as to guide treatment selection. Although there are no commonly applied clinical subtyping strategies or biomarkers to select first- or second-line treatments for

*Correspondence: Dr JH Meyer, CAMH Research Imaging Centre and Campbell Family Mental Health Research Institute, Centre for Addiction and Mental Health, Department of Psychiatry, Pharmacology and Toxicology, Institute of Medical Sciences, University of Toronto, 250 College Street, Toronto, ON M5TIR8, Canada.

Tel. + 4l6-535-850I × 4007, Fax. + 4l6-979-4656;

E-mail: jeff.meyer@camhpet.ca

Received 30 July 20 13; revised 16 October 2013; accepted 17 October 2013; accepted article preview 24 October 2013
$\mathrm{MDE}$, this strategy is anticipated to improve MDE response to treatment because many different biological abnormalities have been identified, including reduced glial cell density, deficient signal transduction, deficient neurogenesis, reduced hippocampal volume, elevated cytokine levels, greater glucocorticoid secretion, greater oxidative stress, markers of increased apoptosis, and indices reflecting lowered monoamines (Meyer, 2012; Rajkowska and Stockmeier, 2013; Schmidt et al, 2011).

Among markers of MDE pathology, there are several key reasons to investigate indices of central monoamine oxidase A (MAO-A) level as a biomarker for subtyping: MAO-A has diverse functions, including facilitating a pro-oxidant state through the production of hydrogen peroxide, participating in apoptosis, influencing mitochondrial function, and metabolizing monoamines (Youdim et al, 2006), each of which have sequelae that may be targeted by a variety of therapeutics. MAO-A level is highly correlated with MAO-A activity in brain (Nelson et al, 1979; Saura et al, 1992) and indices of MAO-A level in the prefrontal (PFC) and anterior cingulate cortex (ACC) were elevated by $25-40 \%$ across the 
three studies that investigated the most common, early onset MDD (Johnson et al, 2011; Meyer et al, 2006; Meyer et al, 2009). Elevated MAO-A level in these regions in MDE is consistent with other specific findings: MDE is a highstress state, and glucocorticoid administration increases transcription rate and catalytic activity of MAO-A in human neuroblastoma and glioblastoma cell lines (Ou et al, 2006a). Furthermore, glucocorticoid administration and/or chronic stress increase MAO-A mRNA and/or MAO-A protein levels in the PFC of rodents (Filipenko et al, 2002; Slotkin et al, 1998). In humans, during MDE, greater whole-brain serotonin turnover has been reported (Barton et al, 2008) and this is consistent with greater MAO-A level and/or activity as MAO-A is the primary route of serotonin metabolism in brain. Also, reduced mRNA and levels of nuclear transcription factor R1, which inhibits MAO-A transcription, and elevated levels of TIEG2, a nuclear transcription factor that promotes transcription of MAO-A, were found in postmortem PFC in MDE (Grunewald et al, 2012; Johnson et al, 2011; Ou et al, 2006a, b).

Additional reasons to pursue clinical subtyping in relation to MAO-A level include the availability of irreversible monoamine oxidase inhibitors (MAOIs) for clinical use and the recent success of new MAOIs that overcome the restriction of dietary tyramine associated with the original MAOIs (Youdim et al, 2006). The latter is best exemplified by the design of compounds with greater selectivity for MAO-A or MAO-B and/or greater reversibility (such as moclobemide and EMSAM) of MAOIs in which the parent medication is inactive peripherally but then metabolized to be active in the brain, thereby increasing the ratio of brain to peripheral concentrations of the drug (Youdim et al, 2006). This latter set of compounds is being tested in phase 2 trials in humans.

In the present study, we apply $\left[{ }^{11} \mathrm{C}\right]$ harmine PET to determine which subtypes of MDE have the highest MAO-A $V_{\mathrm{T}}$, an index of regional brain MAO-A level, with our primary regions of interest being the PFC and ACC (for review of $\left[{ }^{11} \mathrm{C}\right]$ harmine properties see Meyer et al $\left.(2006,2009)\right)$. Although all regions sampled to date tend to demonstrate elevated MAO-A $V_{\mathrm{T}}$ during $\mathrm{MDE}$, we prioritized these two regions because the accumulated evidence is greatest for elevated MAO-A level in these two regions (Johnson et al, 2011; Meyer et al, 2006, 2009) and subregions of these structures are activated during sad mood and negative cognition (Liotti et al, 2002; Ressler and Mayberg, 2007).

For the present study, we focus on two subtypes of MDE: severity (ie, moderate to severe versus mild level of symptoms) and reversed neurovegetative symptoms (presence of hypersomnia and either hyperphagia or weight gain). The rationale for investigating severity was based on a data set from an earlier study in which we observed that in MDE subjects, a score of greater or equal to 20 on the 17-item Hamilton Rating Scale for Depression (HRSD) corresponded to a $15 \%$ elevation in the MAO-A $V_{\mathrm{T}}$ in the PFC relative to those MDE with a HRSD of less than 20 (specific subanalysis not published). The rationale for choosing MDE with reversed neurovegetative symptoms as the second subtype is that these symptoms are associated with a greater likelihood of response to MAOIs (Lonnqvist et al, 1994; Quitkin et al, 1993), which could be explained by greater expression of the MAO-A target, ie, treatments which best match the pathology should have a higher likelihood of response. Moreover, a latent class analysis found that atypical MDE is primarily identified by the symptoms of increased appetite, weight gain, and hypersomnia (Sullivan et al, 1998), and among atypical symptoms, this cluster is associated with the greatest inter-rater reliability (kappa values: hyperphagia, 1.0; hypersomnia, 0.90 ; mood reactivity, 0.83 ; rejection sensitivity, 0.75 ; and leaden paralysis, 0.78; Posternak and Zimmerman, 2001). Thus, the hypotheses of the present study are that subtypes of MDE with greater severity of symptoms (as defined by a HRSD $\geqslant 20$ ) and reversed neurovegetative symptoms will have greater MAO-A $V_{\mathrm{T}}$ in the PFC and ACC.

\section{MATERIALS AND METHODS}

\section{Participants}

Forty-two participants were recruited who met criteria for current MDE and MDD (mean age $=31.6$ years, $\mathrm{SD}=7.5$ ) as determined by the Structured Clinical Interview for DSM-IV Axis I disorders (SCID-I) and verified by subsequent consultation with a psychiatrist (RGC, RDL, or JHM). The presence of reversed neurovegetative symptoms of depression were verified by the SCID-I. Data from 15 subjects had been previously compared with healthy subjects but had not been examined in relation to subtype (and were not the data reviewed in the introduction to justify the first hypothesis). Inclusion criteria were early onset MDD (onset prior to age 45) and current age from 18 to 50 years as the intent of this study was to subtype early onset MDD. Other inclusion criteria were good physical health and a HRSD of at least 14 on the 17-item HRSD. Exclusion criteria included co-morbid psychiatric or medical illness, current or past substance abuse, report of cigarette smoking within the past year (since recent heavy cigarette smoking may bias MAO-A $V_{\mathrm{T}}$ (Bacher et al, 2011; Fowler et al, 1996)), positive urine toxicology drug screen, and herbal drug or medication use within the past 8 weeks with the exception of SSRIs for which the exclusion period was 2 weeks (37 participants had no SSRI use in the past month). Although SSRIs, through reducing extracellular clearance of serotonin, likely counter a downstream effect of greater serotonin metabolism from elevated MAO-A level, we previously demonstrated that SSRIs do not affect MAO-A $V_{\mathrm{T}}$ in vivo (Meyer et al, 2009), a finding consistent with the pharmacological specificity of SSRIs. Specific exclusion criteria for women included current perimenopause, current postmenopause, and a positive urine pregnancy test. Phase of menstrual cycle was recorded by self-report and was consistent with estrogen and progesterone plasma samples obtained. We did not control for phase of menstrual cycle, as in a previously collected sample there was no relationship between phase of menstrual cycle and MAO-A $V_{\mathrm{T}}$ (Meyer et al, 2009). In addition, all participants were screened to rule out borderline and antisocial personality disorder using the Structured Clinical Interview for DSM-IV for Axis II disorders.

Participants were also screened to rule out common medical conditions associated with MDD through plasma sampling for calcium and thyroid-stimulating hormone. Also, participants were required not to consume any caffeine on the day of the PET scan and were required not 
Table I Demographic and Clinical Characteristics of Individuals with Moderate to Severe Depression and Mild to Moderate Depression

\begin{tabular}{lcc}
\hline & $\begin{array}{c}\text { Moderate to severe } \\
\text { depression }(\boldsymbol{n}=\mathbf{2 5})\end{array}$ & $\begin{array}{c}\text { Mild to moderate } \\
\text { depression }(\boldsymbol{n}=\mathbf{I 7})\end{array}$ \\
\hline Age, mean (SD), years & $32.7(7.8)$ & $30.9(7.8)$ \\
HRSD score, mean (SD) & $23.6(2.7)$ & $16.6(2.2)^{\mathrm{a}}$ \\
Age at onset, mean (SD), years & $19.4(9.1)$ & $18.6(10.1)$ \\
No. of previous episodes, mean (SD) & $2.2(1.3)$ & $15(60)$ \\
Female, No. (\%) & $16(64)$ & $10(59)$ \\
Past antidepressant treatment, No. (\%) & $10(59)$ & \\
\hline
\end{tabular}

Abbreviation: HRSD, 17-item Hamilton Rating Scale for Depression.

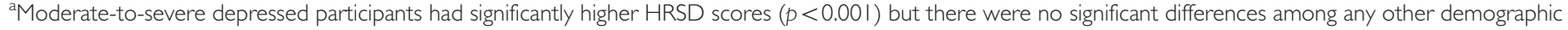
or clinical variables between groups.

to use any over-the-counter medications or consume any alcohol for $48 \mathrm{~h}$ before the PET scan. For a secondary aim of comparing MDE subgroups to health, 37 healthy controls (mean age $=31.9$ years, $\mathrm{SD}=7.6$ ) were recruited. Criteria and screening were identical to those with MDD except all psychiatric illnesses were exclusionary. Demographic characteristics are shown in Tables 1 and 2. After complete description of the study to the subjects, written informed consent was obtained. The study was approved by the Research Ethics Board for Human Subjects at the Centre for Addiction and Mental Health, University of Toronto, in accordance with the Declaration of Helsinki.

\section{Imaging}

Each participant underwent one $\left[{ }^{11} \mathrm{C}\right]$ harmine PET scan to determine MAO-A $V_{\mathrm{T}}$. For each PET scan, $370 \mathrm{MBq}$ of $\left[{ }^{11} \mathrm{C}\right]$ harmine was administered as a bolus intravenously. An automatic blood sampling system was used to measure arterial blood radioactivity over the first $10 \mathrm{~min}$ of the scan. Manual samples were obtained at 2.5, 7.5, 15, 20,30, 45, 60, and $90 \mathrm{~min}$ post injection. The method of measuring radioactivity in whole blood and parent compound in plasma has been previously described (Ginovart et al, 2006). PET images were acquired using an HRRT PET camera (in-plane resolution; full-width half-maximum, $3.1 \mathrm{~mm} ; 207$ axial sections of $1.2 \mathrm{~mm}$; Siemens Molecular Imaging, Knoxville, TN, USA) as previously described (Meyer et al, 2009). The frames consisted of 15 frames of 1 min followed by 15 frames of $5 \mathrm{~min}$. $\left[{ }^{11} \mathrm{C}\right]$ Harmine doses were of high specific activity (mean: $1572.60 \mathrm{mCi} / \mu \mathrm{mol}, \mathrm{SD}: 1024.61 \mathrm{mCi} / \mu \mathrm{mol}$ ) and high radiochemical purity (mean: $98.7 \%$, SD: $0.95 \%$ ).

Each participant also underwent magnetic resonance imaging (GE Signa 1.5-T scanner; fast spoiled gradient echo, $\mathrm{T}_{1}$-weighted image; $x, y, z$ voxel dimensions, 0.78 , 0.78 , and $1.5 \mathrm{~mm}, \mathrm{GE}$ Medical Systems, Milwaukee, WI, USA) for the region of interest (ROI) delineation. The ROIs were determined using a semi-automated method in which regions of a template MRI are transformed on to the individual MRI based on a series of transformations and deformations that match the template image to the individual co-registered MRI as well as segmentation of the individual MRI to select the gray matter voxels as previously described (Meyer et al, 2009; Rusjan et al, 2006).
The primary ROIs were the PFC and ACC because these regions (and their subregions) participate in the affective regulation as demonstrated by paradigms of mood induction and affective cognition (Liotti et al, 2002; Ressler and Mayberg, 2007) Secondary ROIs included regions that are implicated in MDE and/or have moderate to high MAO-A density and included hippocampus, ventral striatum, dorsal putamen, thalamus, and midbrain. In addition, several subregions of the prefrontal cortex were sampled, including dorsolateral prefrontal cortex (DLPFC), ventrolateral prefrontal cortex (VLPFC), medial prefrontal cortex (mPFC), and orbitofrontal cortex (OFC). The borders of the subregions of the PFC were defined based on their cytoarchitectural differences from adjacent cortex (Rajkowska and Goldman-Rakic, 1995a, b; Uylings et al, 2010).

MAO-A $V_{\mathrm{T}}$ represents the total distribution volume of $\left[{ }^{11} \mathrm{C}\right]$ harmine and it is an index of tissue binding at equilibrium, of which $85 \%$ is specific binding to MAO-A, for further details see the Supplementary Methods section. Therefore, differences in MAO-A $V_{\mathrm{T}}$ may be interpreted as representing changes in $\left[{ }^{11} \mathrm{C}\right]$ harmine binding to MAO-A. The $V_{\mathrm{T}}$ can be expressed in terms of kinetic rate parameters of a two-tissue compartment model: $V_{\mathrm{T}}=\left(K_{1} / k_{2}\right) \times\left(k_{3} /\right.$ $\left.k_{4}\right)+\left(K_{1} / k_{2}\right)$, where $K_{1}$ and $k_{2}$ are the influx and efflux rate constants for radiotracer passage across the blood-brain barrier and $k_{3}$ and $k_{4}$ describe the radioligand transfer between the free and nonspecific compartment and the specific binding compartment. The ratio of $K_{1}$ to $K_{2}$ is similar among different individuals (for further detail, see Ginovart et al (2006)). For $\left[{ }^{11} \mathrm{C}\right]$ harmine PET, $V_{\mathrm{T}}$ may be validly and reliably measured with either an unconstrained two-tissue compartment model or with the Logan model with arterial sampling (for which the underestimate of $V_{\mathrm{T}}$ is negligible at the noise level of time activity curves from the regions of interest; Ginovart et al, 2006), and the latter was applied in this study. This method has been previously described in detail (Ginovart et al, 2006; Meyer et al, 2009).

\section{Statistical Analyses}

The primary analysis, was a multivariate analysis of variance (MANOVA) with predictor variables of severity (greater or equal to 20 on the 17-item HRSD) and reversed neurovegetative symptoms (both hypersomnia and either hyperphagia or weight gain) and the dependent variables 
Table 2 Demographic and Clinical Characteristics Data of Individuals with and without Reversed Neurovegetative Symptoms of Depression $^{\mathrm{a}}$

\begin{tabular}{lcc}
\hline & $\begin{array}{c}\text { With reversed neurovegetative } \\
\text { symptoms } \\
(\mathbf{n = 1 0 )}\end{array}$ & $\begin{array}{c}\text { Without reversed neurovegetative } \\
\text { symptoms } \\
(\mathbf{n =}=\mathbf{3 2})\end{array}$ \\
\hline Age, mean (SD), years & $31.3(9.0)$ & $32.2(7.5)$ \\
HRSD score, mean (SD) & $18.8(2.9)$ & $21.4(4.4)$ \\
Age at onset, mean (SD), years & $16.5(6.8)$ & $19.9(10.0)$ \\
No. of previous episodes, mean (SD) & $2.4(1.3)$ & $2.4(1.6)$ \\
Female, No. (\%) & $5(50)$ & $20(63)$ \\
Past antidepressant treatment, No. (\%) & $8(80)$ & $18(56)$ \\
\hline
\end{tabular}

Abbreviation: HRSD, 17-item Hamilton Rating Scale for Depression.

a There were no significant differences among any of the demographic or clinical variables between groups.

were MAO-A $V_{\mathrm{T}}$ in the PFC and ACC. A secondary analysis used a MANOVA applying the same predictor variables, however, the dependent variables were MAO-A $V_{\mathrm{T}}$ in all brain regions (to assess whether the predictor variables related to MAO-A $V_{\mathrm{T}}$ globally in the brain). Other secondary analyses applied MANOVA to compare MAO-A $V_{\mathrm{T}}$ in the PFC and ACC between MDE subgroups and the healthy group. The four subgroupings assessed were moderate-to-high severity, mild-to-moderate severity, reversed neurovegetative symptoms present, and reversed neurovegetative symptoms absent.

\section{RESULTS}

\section{Effect of Severity upon MAO-A $\mathrm{V}_{\mathrm{T}}$}

There was a main effect of severity on MAO-A $V_{\mathrm{T}}$ in the PFC and ACC (MANOVA, $\mathrm{F}_{(2,38)}=5.44, p=0.008$ ) and in the subregions of the PFC (OFC, VLPFC, DLPFC, mPFC; MANOVA, $\left.\mathrm{F}_{(4,36)}=4.57, p=0.004\right)$. The whole-brain analysis did not reach significance (MANOVA, $\mathrm{F}_{(7,33)}=1.90$, $p=0.10$ ) but there was a tendency toward a similar finding across all regions sampled (individual ANOVA, $\left.\mathrm{F}_{(1,39)}=5.31-11.01, \quad p=0.03-0.002\right)$, and a trend in the hippocampus (ANOVA, $\mathrm{F}_{(1,39)}=2.59, p=0.1$; Figure 1 ).

\section{Effect of Reversed Neurovegetative Symptoms upon MAO-A $V_{\mathrm{T}}$}

All of the subjects enrolled who had hypersomnia also had either hyperphagia or weight gain (and vice versa). After including the effect of severity, there was also an effect of reversed neurovegetative symptoms on MAO-A $V_{\mathrm{T}}$ in the PFC and ACC (MANOVA, $\mathrm{F}_{(2,38)}=5.13, p=0.01$ ), which was also present in subregions of the PFC (OFC, VLPFC, DLPFC, mPFC; MANOVA, $\left(\mathrm{F}_{(4,36)}=3.23, p=0.02\right)$. There was a similar tendency across all regions sampled (MANOVA, $_{(7,33)}=2.56, p=0.03$; Figure 2$)$.

\section{Relationship of MAO-A $V_{\mathrm{T}}$ with Severity as a Continuous Variable}

Applying a multivariate analysis of co-variance (MANCOVA) with the dependent variables being MAO-A $V_{\mathrm{T}}$ in the
PFC and ACC, and predictor of HDRS as a covariate and reversed neurovegetative features as a factor, we found both to be significant predictors (severity: $\mathrm{F}_{2,38}=5.09, p=0.01$, reversed neurovegetative symptoms $\mathrm{F}_{2,38}=4.71, p=0.02$ ). ANOVAs within these two regions, with MAO-A $V_{\mathrm{T}}$ as the dependent variable, were also significant (PFC: severity, $\mathrm{F}_{1,39}=10.44, \quad p=0.003$, reversed neurovegetative symptoms, $F_{1,39}=8.04, p=0.007$; ACC: severity, $\mathrm{F}_{1,39}=8.26, p=0.007$, reversed neurovegetative symptoms, $\left.\mathrm{F}_{1,39}=9.64, p=0.004\right)$.

\section{Comparison of MAO-A $V_{\mathrm{T}}$ between Subgroups with Healthy Controls}

Participants with moderate-to-severe $\operatorname{MDE}(n=25)$ had significantly greater MAO-A $V_{\mathrm{T}}$ in the PFC and ACC compared with healthy controls $(n=37$; MANOVA, $\left.\mathrm{F}_{(2,59)}=9.75, p<0.001\right)$ and this was also found in all brain regions (MANOVA, $\mathrm{F}_{(7,54)}=3.41, p=0.004$ ). The subgroup with reversed neurovegetative symptoms $(n=10)$ also had significantly greater MAO-A $V_{\mathrm{T}}$ in the PFC and ACC compared with controls (MANOVA, $\mathrm{F}_{(2,44)}=5.710$, $p<0.01)$, which was also found across all brain regions (MANOVA, $\left.\mathrm{F}_{(7,39)}=3.32, p=0.007\right)$. Participants without reversed neurovegetative symptoms $(n=32)$ of depression also had significantly greater MAO-A $V_{\mathrm{T}}$ in the PFC and ACC relative to controls (MANOVA $\mathrm{F}_{(2,66)}=5.71, p<0.01$ ), but subjects with mild-to-moderate $\operatorname{MDE}(n=17)$ had no significant difference in MAO-A $V_{\mathrm{T}}$ in the PFC and ACC relative to controls (MANOVA, $\mathrm{F}_{(2,51)}=1.94, p=0.16$ ).

\section{DISCUSSION}

This is the first study to investigate the relationship between symptoms of MDE and MAO-A $V_{\mathrm{T}}$. The main findings are that MAO-A $V_{\mathrm{T}}$ is significantly greater in the PFC and ACC in moderate-to-severe $\mathrm{MDE}$ and in MDE with reversed neurovegetative symptoms. Although we focused on the PFC and ACC, there was a tendency for a similar association in all the brain regions sampled. The ability to identify the subtype of MDE with the highest level of MAO-A $V_{\mathrm{T}}$ offers an important opportunity to improve the development and application of therapeutics that target MAO-A or the 


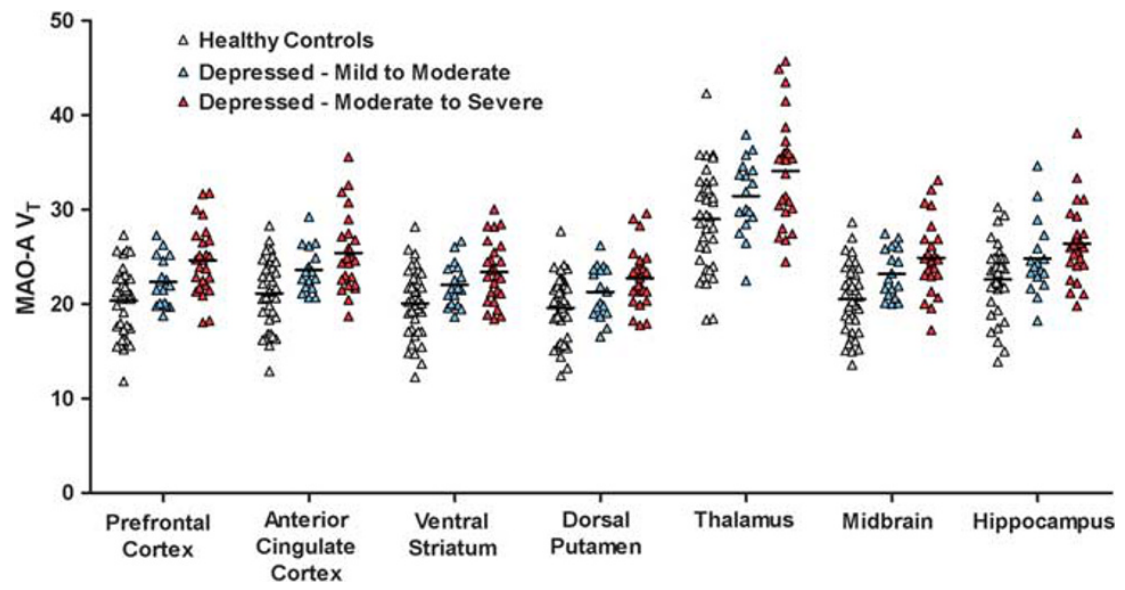

Figure I The effect of moderate to severe symptoms of depression (HRSD $\geqslant 20$ ) compared with mild to moderate symptoms (HRSD $\leqslant 19$ ) of depression on MAO-A $V_{T}$ in regions of interest. Participants with severe depression had significantly higher MAO-A $V_{T}$ in the prefrontal and anterior cingulate cortices (multivariate analysis of variance (MANOVA), $F_{(2,38)}=5.44, p=0.008$ ). The whole-brain analysis did not reach significance (MANOVA, $\left.F_{(7,33)}=1.90, p=0.10\right)$ but there was a tendency toward a similar finding in the other regions sampled (individual $A N O V A, F(1,39)=5.3 \mid$ to $\mid I .0 I$, $p=0.002-0.03$ ), and a trend in the hippocampus (ANOVA, $F_{(1,39)}=2.59, p=0.1$ ).

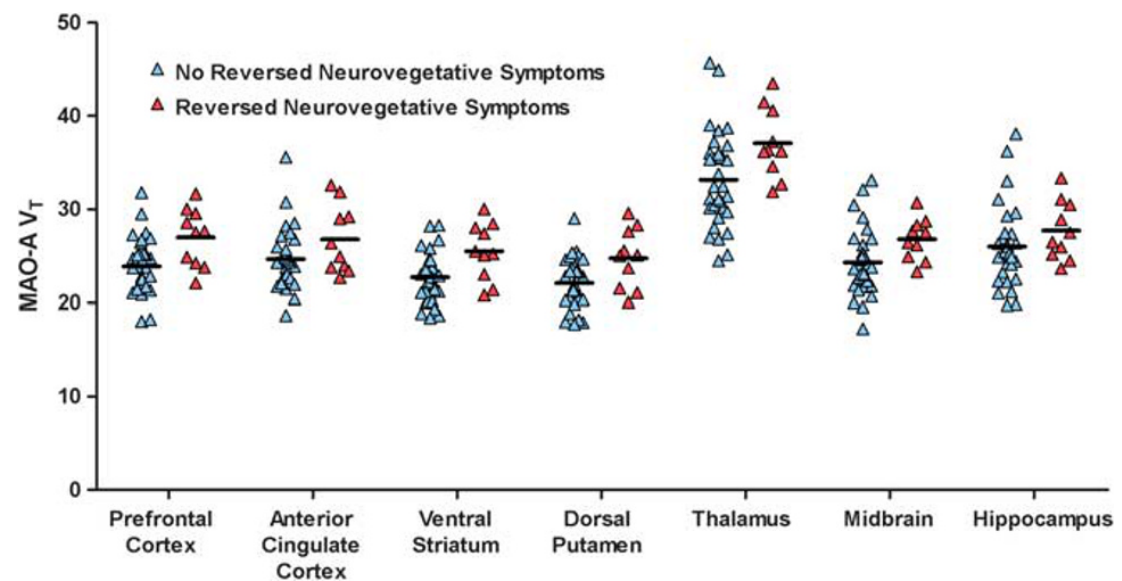

Figure 2 The effect of reversed neurovegetative symptoms of depression compared with no reversed neurovegetative symptoms of depression on $\mathrm{MAO}-\mathrm{A} V_{T}$ in regions of interest. Participants with reversed neurovegetative symptoms of depression had significantly higher $M A O-A V_{T}$ in the prefrontal and anterior cingulate cortices (multivariate analysis of variance (MANOVA), $F_{(2,38)}=5.13, p=0.01$ ) and this effect was also present across all the brain regions sampled (MANOVA, $F_{(7,33)}=2.56, p=0.03$ ). To visually demonstrate the effect of reversed neurovegetative symptoms of depression on $M A O-A$ $V_{T}$, the variance due to severity was removed from the MAO-A $V_{T}$ values in this plot. The difference in mean MAO-A $V_{T}$ value between low- and high-severity subtypes was added to the low-severity subtypes so severity no longer contributed to the variance.

sequelae of elevated MAO-A level. In addition, these findings suggest new mechanistic relationships between MAO-A level and the symptom clusters of severity and reversed neurovegetative symptoms.

As optimal matching of therapeutic to illness pathology should increase the likelihood of response, the association between greater severity and reversed neurovegetative symptoms with higher MAO-A $V_{\mathrm{T}}$ in the PFC and ACC has the potential to be applied to improve treatments that target functions of MAO-A, or the sequelae of elevated MAO-A level. Treatments that counter functions of MAO-A include not only MAO inhibitors, but also several medication classes in development such as multiple monoamine reuptake inhibitors, and neuroprotective medications with antioxidant, anti-apoptotic or mitochondrial protecting properties. To date, among the symptoms correlated with elevated MAO-A $V_{\mathrm{T}}$, the best evidence for a relationship to clinical response is the association between reversed neurovegetative symptoms with response to MAOIs (Lonnqvist et al, 1994; McGrath et al, 1993; Quitkin et al, 1993). However, greater global severity of MDE symptoms has also been associated with greater likelihood of MAOI response (Thase et al, 1992). Although the current findings argue in support of clinical trials investigating the relationship between these symptom clusters and response to specific types of antidepressants, they also have implications for the general development of these classes of medications for phase 2 and 3 trials. Approximately 50\% of clinical trials with marketed antidepressants fail to differentiate between the active therapeutic and placebo, hence, it would be reasonable to ensure that future trials of medications targeting elevated MAO-A level or the sequelae of elevated MAO-A level sample a reasonable proportion of participants with a high severity of symptoms and/or reversed neurovegetative symptoms. It is therefore possible that a clinical trial with a sample poor in these subtypes 
could, on average, have pathology that may be a poorer match to the therapeutic, and therefore be less likely to achieve a superior response to placebo.

We suggest that the most likely explanation for the association between greater MAO-A $V_{\mathrm{T}}$ in the PFC and ACC and greater severity is that elevated MAO-A level in these regions leads to a greater severity of symptoms. In brain tissue, MAO-A level is highly correlated with MAO-A activity and MAO-A $V_{\mathrm{T}}$ is primarily an index of MAO-A level (Saura et al, 1992). Elevated MAO-A activity is related to several important downstream effects, as MAO-A participates in the metabolism of serotonin, dopamine, and norepinephrine, produces the pro-oxidant hydrogen peroxide and influences the predisposition toward apoptosis (Fitzgerald et al, 2007; Ou et al, 2006b; Youdim et al, 2006). The specific regions assayed in this study, which have a reasonably high MAO-A density, also participate in neural systems dysregulated during MDE and elevations of MAO-A $V_{\mathrm{T}}$ in these regions could potentially account for the individual symptoms of MDE. Specifically, subregions of the PFC and ACC are active during the production of sad mood induction and the anticipation of negative events (Liotti et al, 2002; Ressler and Mayberg, 2007); dorsal striatum influences executive function and psychomotor speed; ventral striatum influences the anticipation of reward (O'Doherty et al, 2004) and the midbrain contains monoaminergic nuclei that control the onset and maintenance of sleep (Kajimura et al, 1999). Hence, the locations of excessively elevated MAO-A $V_{\mathrm{T}}$ have the potential to influence many of the neural systems that function abnormally during MDE, and thereby influence illness severity. Empirically, chronic depletion of multiple monoamines through reserpine administration, or acute depletion of subsets of monoamines like serotonin through tryptophan depletion, or norepinephrine and dopamine through alphamethylparatyrosine administration is associated with sad mood in humans (Freis, 1954; Verhoeff et al, 2003; Young et al, 1985). Oxidative stress elicited through overexpression of glyoxalase 1 and glutathione reductase 1 genes or through other methods such as xanthine and xanthine oxidase treatment is associated with anxiety behavior in preclinical models (Hovatta et al, 2005; Salim et al, 2010). Genetic vulnerability toward apoptosis is associated with greater risk of MDD in some human subpopulations (Harlan et al, 2006) and both mRNA and protein levels of R1 (a nuclear transcription factor which facilitates resistance against apoptosis) are reduced in the PFC in MDD (Johnson et al, 2011; Thalmeier et al, 2008).

To the best of our knowledge, the present study represents the first example of an abnormality in MDE that is increased in the presence of reversed neurovegetative symptoms. Most biological markers of $\mathrm{MDE}$, such as increased plasma TNF $\alpha$ levels, and hypothalamic-pituitary-axis-cortisol activity, are suppressed when reversed neurovegetative symptoms are present (Dunjic-Kostic et al, 2012; Gold and Chrousos, 2002; Karlovic et al, 2012). Thus, the association between elevated MAO-A $V_{\mathrm{T}}$ in the PFC and ACC and reversed neurovegetative symptoms provides new directions to investigate their etiology, particularly for weight gain. A number of factors may lead to elevated MAO-A level or activity, including glucocorticoid administration, estrogen depletion and mitochondrial toxicity/ dysfunction (Fitzgerald et al, 2007; Ou et al, 2006a; Sacher et al, 2010). We suggest that the latter marker, if present throughout the body and brain, may be the best theoretical direction to investigate for weight gain because mitochondrial dysfunction in muscle has been identified in obesity and impaired mitochondrial function reduces the ability of muscle to be active and expend energy (Lowell and Shulman, 2005; Patti and Corvera, 2010).

The current study has the advantage of measuring an index of MAO-A density in vivo but has disadvantages inherent to PET imaging. The resolution of PET does not allow investigation of the cellular specificity of the changes in MAO-A $V_{\mathrm{T}}$ as MAO-A can be present in both glia and neurons. Even so, given that the most likely mechanism for elevated MAO-A in MDD is excessive glucocorticoid secretion, and glucocorticoids promote transcription of MAO-A in both of these general cell types, it is likely that severity is related to MAO-A level in both cell types. In addition, levels of the transcription factors implicated in greater MAO-A expression in MDD, such as reduced expression of R1 (a MAO-A repressor) and greater expression of $\mathrm{Sp} 1$ (a MAO-A activator), are similarly modulated by glucocorticoids in both glia and neurons (Ou et al, 2006a). A second limitation is that we selected MAO-A $V_{\mathrm{T}}$ as a marker. The advantage of this marker is that it is robustly measured with $\left[{ }^{11} \mathrm{C}\right]$ harmine PET, but the disadvantage is that it represents both specific and free and nonspecific binding. Nevertheless, the free and nonspecific binding for $\left[{ }^{11} \mathrm{C}\right]$ harmine represents only $15 \%$ of MAO-A $V_{\mathrm{T}}$ so the difference in this measure primarily reflects a change in specific MAO-A binding.

In conclusion, greater MAO-A $V_{\mathrm{T}}$ in the PFC and ACC is associated with greater global severity of symptoms and reversed neurovegetative symptoms. A great asset of this work is that these symptoms are straightforward to measure clinically and it is rare for clinical markers of MDE to be strongly associated with biological markers of MDE. Based on the principle that optimal matching of treatment to pathology should result in a greater therapeutic response, these findings can be applied to improve sampling of patients for phase 2 and phase 3 trials for treatments that specifically target MAO-A or the sequelae of elevated MAOA. In addition, the findings unveil new directions toward understanding the symptoms of MDE, suggesting either a causal link (such as the association of greater MAO-A level in the PFC and ACC with greater severity of symptoms) or a correlative link (such as a marker of associated pathology in the case of the reversed neurovegetative symptoms). Finally, these results are important as it has been over 25 years since it was known that reversed neurovegetative symptoms are more likely to respond to MAOI (Lonnqvist et al, 1994; Quitkin et al, 1993), and greater MAO-A expression represents the first explanation that involves identification of a matching biological abnormaility in MDE, which is exacerbated when reversed neurovegetative symptoms are present.

\section{FUNDING AND DISCLOSURE}

This work was supported by grants from the Canadian Institutes of Health Research (CIHR) and salary support from the Ontario Mental Health Foundation and CIHR. 
Drs. Meyer, Wilson, and Houle have received operating grant funding for other studies from Eli Lilly, GlaxoSmithKline, Bristol-Myers Squibb, Lundbeck, and SK Life Science in the past 5 years. Dr Meyer has consulted to several of these companies as well as Sepracor, Trius Therapeutics and Mylan Inc. None of these companies participated in the funding, design or execution of this study or writing the manuscript. Dr Meyer is developing natural health products to treat high MAO-A states. Dr Meyer is also applying for patents to implement measures of utilizing MAO to diagnose or treat mood disorders. It is likely that companies which make medications affecting monoamine receptor or monoamine oxidase binding will seek collaborations with these investigators in the future. Dr Kish receives research funding from US NIH NIDA DA25096 and a salary from the Centre for Addiction and Mental Health. The authors declare no conflict of interest.

\section{ACKNOWLEDGEMENTS}

We thank the Campbell Family Mental Health Research Institute; administrative assistant Natasha Bennett; technicians Alvina $\mathrm{Ng}$ and Laura Nguyen; chemistry staff Jun Parkes, Armando Garcia, Winston Stableford, and Min Wong; engineers Terry Bell and Ted Harris-Brandts; and students Shakira Mohammed and Kimberly De Sousa for their assistance with this project. Dr Meyer takes responsibility for the integrity of the data and the accuracy of the data analysis, and that all authors had full access to all the data in the study.

\section{REFERENCES}

Bacher I, Houle S, Xu X, Zawertailo L, Soliman A, Wilson AA et al (2011). Monoamine oxidase A binding in the prefrontal and anterior cingulate cortices during acute withdrawal from heavy cigarette smoking. Arch General Psychiatry 68: 817-826.

Barton DA, Esler MD, Dawood T, Lambert EA, Haikerwal D, Brenchley C et al (2008). Elevated brain serotonin turnover in patients with depression: effect of genotype and therapy. Arch General Psychiatry 65: 38-46.

Dunjic-Kostic B, Ivkovic M, Radonjic NV, Petronijevic ND, Pantovic M, Damjanovic A et al (2012). Melancholic and atypical major depression-Connection between cytokines, psychopathology and treatment. Prog Neuropsychopharmacol Biol Psychiatry 43C: 1-6.

Filipenko ML, Beilina AG, Alekseyenko OV, Dolgov VV, Kudryavtseva NN (2002). Repeated experience of social defeats increases serotonin transporter and monoamine oxidase A mRNA levels in raphe nuclei of male mice. Neurosci Lett 321: 25-28.

Fitzgerald JC, Ufer C, De Girolamo LA, Kuhn H, Billett EE (2007). Monoamine oxidase-A modulates apoptotic cell death induced by staurosporine in human neuroblastoma cells. J Neurochem 103: 2189-2199.

Fowler JS, Volkow ND, Wang GJ, Pappas N, Logan J, Shea C et al (1996). Brain monoamine oxidase A inhibition in cigarette smokers. Proc Natl Acad Sci USA 93: 14065-14069.

Freis ED (1954). Mental depression in hypertensive patients treated for long periods with large doses of reserpine. $N$ Engl J Med 251: 1006-1008.

Ginovart N, Meyer JH, Boovariwala A, Hussey D, Rabiner EA, Houle $S$ et al (2006). Positron emission tomography quantification of [11C]-harmine binding to monoamine oxidase-A in the human brain. J Cereb Blood Flow Metab 26: 330-344.
Gold PW, Chrousos GP (2002). Organization of the stress system and its dysregulation in melancholic and atypical depression: high vs low CRH/NE states. Mol Psychiatry 7: 254-275.

Grunewald M, Johnson S, Lu D, Wang Z, Lomberk G, Albert PR et al (2012). Mechanistic role for a novel glucocorticoid-KLF11 (TIEG2) protein pathway in stress-induced monoamine oxidase A expression. J Biol Chem 287: 24195-24206.

Harlan J, Chen Y, Gubbins E, Mueller R, Roch JM, Walter K et al (2006). Variants in Apaf-1 segregating with major depression promote apoptosome function. Mol Psychiatry 11: 76-85.

Hovatta I, Tennant RS, Helton R, Marr RA, Singer O, Redwine JM et al (2005). Glyoxalase 1 and glutathione reductase 1 regulate anxiety in mice. Nature 438: 662-666.

Johnson S, Stockmeier CA, Meyer JH, Austin MC, Albert PR, Wang $\mathrm{J}$ et al (2011). The reduction of R1, a novel repressor protein for monoamine oxidase a, in major depressive disorder. Neuropsychopharmacology 36: 2139-2148.

Kajimura N, Uchiyama M, Takayama Y, Uchida S, Uema T, Kato M et al (1999). Activity of midbrain reticular formation and neocortex during the progression of human non-rapid eye movement sleep. J Neurosci 19: 10065-10073.

Karlovic D, Serretti A, Vrkic N, Martinac M, Marcinko D (2012). Serum concentrations of CRP, IL-6, TNF-alpha and cortisol in major depressive disorder with melancholic or atypical features. Psychiatry research 198: 74-80.

Liotti M, Mayberg HS, McGinnis S, Brannan SL, Jerabek P (2002). Unmasking disease-specific cerebral blood flow abnormalities: mood challenge in patients with remitted unipolar depression. Am J Psychiatry 159: 1830-1840.

Lonnqvist J, Sihvo S, Syvalahti E, Kiviruusu O (1994). Moclobemide and fluoxetine in atypical depression: a double-blind trial. J Affect Disord 32: 169-177.

Lowell BB, Shulman GI (2005). Mitochondrial dysfunction and type 2 diabetes. Science 307: 384-387.

McGrath PJ, Stewart JW, Nunes EV, Ocepek-Welikson K, Rabkin JG, Quitkin FM et al (1993). A double-blind crossover trial of imipramine and phenelzine for outpatients with treatmentrefractory depression. Am J Psychiatry 150: 118-123.

Meyer JH (2012). Neuroimaging markers of cellular function in major depressive disorder: implications for therapeutics, personalized medicine, and prevention. Clin Pharmacol Ther 91: 201-214.

Meyer JH, Ginovart N, Boovariwala A, Sagrati S, Hussey D, Garcia A et al (2006). Elevated monoamine oxidase a levels in the brain: an explanation for the monoamine imbalance of major depression. Arch General Psychiatry 63: 1209-1216.

Meyer JH, Wilson AA, Sagrati S, Miler L, Rusjan P, Bloomfield PM et al (2009). Brain monoamine oxidase A binding in major depressive disorder: relationship to selective serotonin reuptake inhibitor treatment, recovery, and recurrence. Arch General Psychiatry 66: 1304-1312.

Nelson DL, Herbet A, Glowinski J, Hamon M (1979). [3H]Harmaline as a specific ligand of MAO A-II. Measurement of the turnover rates of MAO A during ontogenesis in the rat brain. J Neurochem 32: 1829-1836.

O’Doherty J, Dayan P, Schultz J, Deichmann R, Friston K, Dolan RJ (2004). Dissociable roles of ventral and dorsal striatum in instrumental conditioning. Science 304: 452-454.

Ou XM, Chen K, Shih JC (2006a). Glucocorticoid and androgen activation of monoamine oxidase A is regulated differently by $\mathrm{R} 1$ and Sp1. J Biol Chem 281: 21512-21525.

Ou XM, Chen K, Shih JC (2006b). Monoamine oxidase A and repressor R1 are involved in apoptotic signaling pathway. Proc Natl Acad Sci USA 103: 10923-10928.

Patti ME, Corvera S (2010). The role of mitochondria in the pathogenesis of type 2 diabetes. Endocr Rev 31: 364-395.

Posternak MA, Zimmerman M (2001). Symptoms of atypical depression. Psychiatry Res 104: 175-181. 
Quitkin FM, Stewart JW, McGrath PJ, Tricamo E, Rabkin JG, OcepekWelikson K et al (1993). Columbia atypical depression. A subgroup of depressives with better response to MAOI than to tricyclic antidepressants or placebo. Br J Psychiatry Suppl 21: 30-34.

Rajkowska G, Goldman-Rakic PS (1995a). Cytoarchitectonic definition of prefrontal areas in the normal human cortex: I. Remapping of areas 9 and 46 using quantitative criteria. Cereb Cortex 5: 307-322.

Rajkowska G, Goldman-Rakic PS (1995b). Cytoarchitectonic definition of prefrontal areas in the normal human cortex: II. Variability in locations of areas 9 and 46 and relationship to the Talairach Coordinate System. Cereb Cortex 5: 323-337.

Rajkowska G, Stockmeier CA (2013). Astrocyte pathology in major depressive disorder: insights from human postmortem brain tissue. Curr Drug Targets 14: 1225-1236.

Ressler KJ, Mayberg HS (2007). Targeting abnormal neural circuits in mood and anxiety disorders: from the laboratory to the clinic. Nat Neurosci 10: 1116-1124.

Rusjan P, Mamo D, Ginovart N, Hussey D, Vitcu I, Yasuno F et al (2006). An automated method for the extraction of regional data from PET images. Psychiatry Res 147: 79-89.

Sacher J, Wilson AA, Houle S, Rusjan P, Hassan S, Bloomfield PM et al (2010). Elevated brain monoamine oxidase A binding in the early postpartum period. Arch General Psychiatry 67: 468-474.

Salim S, Asghar M, Chugh G, Taneja M, Xia Z, Saha K (2010). Oxidative stress: a potential recipe for anxiety, hypertension and insulin resistance. Brain Res 1359: 178-185.

Saura J, Kettler R, Da Prada M, Richards JG (1992). Quantitative enzyme radioautography with 3H-Ro 41-1049 and 3H-Ro 196327 in vitro: localization and abundance of MAO-A and MAO-B in rat CNS, peripheral organs, and human brain. J Neurosci 12: 1977-1999.

Schmidt HD, Shelton RC, Duman RS (2011). Functional biomarkers of depression: diagnosis, treatment, and pathophysiology. Neuropsychopharmacology 36: 2375-2394.
Slotkin TA, Seidler FJ, Ritchie JC (1998). Effects of aging and glucocorticoid treatment on monoamine oxidase subtypes in rat cerebral cortex: therapeutic implications. Brain Res Bull 47: 345-348.

Sullivan PF, Kessler RC, Kendler KS (1998). Latent class analysis of lifetime depressive symptoms in the national comorbidity survey. Am J Psychiatry 155: 1398-1406.

Thalmeier A, Dickmann M, Giegling I, Schneider B, M Hartmann A, Maurer K et al (2008). Gene expression profiling of post-mortem orbitofrontal cortex in violent suicide victims. Int J Neuropsychopharmacol 11: 217-228.

Thase ME, Frank E, Mallinger AG, Hamer T, Kupfer DJ (1992). Treatment of imipramine-resistant recurrent depression, III: Efficacy of monoamine oxidase inhibitors. J Clin Psychiatry 53: 5-11.

Trivedi MH, Rush AJ, Wisniewski SR, Nierenberg AA, Warden D, Ritz L et al (2006). Evaluation of outcomes with citalopram for depression using measurement-based care in $S T A R^{\star} D$ : implications for clinical practice. Am J Psychiatry 163: 28-40.

Uylings HB, Sanz-Arigita EJ, de Vos K, Pool CW, Evers P, Rajkowska G (2010). 3-D cytoarchitectonic parcellation of human orbitofrontal cortex correlation with postmortem MRI. Psychiatry Res 183: 1-20.

Verhoeff NP, Christensen BK, Hussey D, Lee M, Papatheodorou G, Kopala L et al (2003). Effects of catecholamine depletion on D2 receptor binding, mood, and attentiveness in humans: a replication study. Pharmacol Biochem Behav 74: 425-432.

WHO (2008): The Global Burden of Disease: 2004 UpdateWorld Health Organization.

Youdim MB, Edmondson D, Tipton KF (2006). The therapeutic potential of monoamine oxidase inhibitors. Nat Rev Neurosci 7: 295-309.

Young SN, Smith SE, Pihl RO, Ervin FR (1985). Tryptophan depletion causes a rapid lowering of mood in normal males. Psychopharmacology (Berl) 87: 173-177.

Supplementary Information accompanies the paper on the Neuropsychopharmacology website (http://www.nature.com/npp) 